\title{
Reasons for early readmission after percutaneous nephrolithotomy and retrograde intrarenal surgery
}

\author{
Sarp Korcan Keskin ${ }^{1}$, Yavuz Onur Danacioglu ${ }^{2}$, Turgay Turan², Ramazan Gokhan Atis $^{2}$, Cengiz Canakci², \\ Turhan Caskurlu², Ali Erol², Asif Yildirim² \\ ${ }^{1}$ Department of Urology, Bahçeşehir University, Istanbul, Turkey \\ ${ }^{2}$ Department of Urology, Istanbul Medeniyet University, Istanbul, Turkey
}

Videosurgery Miniinv 2019; 14 (2): 271-277

DOI: https://doi.org/10.5114/wiitm.2018.77705

\begin{abstract}
Introduction: Hospital readmissions are frequent and costly. In many countries health governors focus on unplanned postsurgical hospital readmissions as an objective metric for quality of care.

Aim: To investigate the rate of readmissions after retrograde intrarenal surgery (RIRS) and percutaneous nephrolithotomy (PCNL) operations, classify these factors, define the higher risk patients for readmission and develop prevention strategies.

Material and methods: A historical cohort study was conducted for a period of 36 months, between 2013 and 2016. A total of 471 consecutive patients, of whom 177 had PCNL (37.6\%) and the remaining 294 had RIRS (62.4\%), were included. The two groups were compared in terms of stone burden, previous stone treatments, initial symptoms, ASA class, intra-operative complications, post-operative stenting, and drugs prescribed at discharge, so as to find the factors influencing the readmission rate for both groups.

Results: The PCNL operation was found to have a significantly higher risk for readmission when compared to RIRS (27.1\% vs. $20.4 \%$, respectively, $p=0.0041$ ). Perioperative complications ( $p=0.002$ for PCNL and $p=0.001$ for RIRS), residual stone(s) or fragments after the operation ( $p=0.002$ for PCNL and $p=0.001$ for RIRS) significantly increased the readmission rate in both groups. The readmission rates were individually affected by postoperative JJ stent placement in the PCNL group ( $p=0.001)$ and previous stone treatments for the RIRS group $(p=0.001)$.

Conclusions: Readmission rates were higher in the PCNL group, but the influencing factors were similar for both groups. The presence of multiple stones preoperatively and residual stones or fragments postoperatively are the most important risk factors for early re-admission after PCNL and RIRS.
\end{abstract}

Key words: retrograde intrarenal surgery, percutaneous nephrolithotomy, kidney stone, readmission, nephrolithiasis.

\section{Introduction}

Retrograde intrarenal surgery (RIRS) has been increasingly used for the surgical management of upper urinary tract stones. With the development of surgical instruments with improved deflection mechanisms, visibility, and durability, the role of RIRS has expanded to the treatment of urinary calculi located in the upper urinary tract, which obviates the shortcomings of shock wave lithotripsy and conventional ureteroscopy $[1,2]$.

Since its initial introduction in 1976, percutaneous nephrolithotomy (PCNL) has been widely performed in the management of large renal stones. There have been a number of subsequent modifications for making this surgery less invasive, such as

\section{Address for correspondence}

Sarp Korcan Keskin MD, Department of Urology, Bahçeşehir University, 34720 Istanbul, Turkey, phone: +90 5359444400 ,

e-mail: sarpkorcan.keskin@med.bau.edu.tr 
tubeless PCNL and mini-micro PCNL $[3,4]$. However, except for open and laparoscopic surgery, PCNL is still the most invasive stone surgery technique [5]. Increasing experience as well as improvements of the RIRS equipment have led to a decrease of PCNL frequency over time, due to its relatively higher complication rates. However, PCNL is currently recommended for staghorn calculi, kidney stones larger than $2 \mathrm{~cm}$, and shock wave lithotripsy-resistant lower pole stones greater than $1 \mathrm{~cm}$ [6].

Readmission is defined as an admission to a hospital within 30 days of a discharge from the same or another hospital due to related health problems. Hospital readmissions are frequent and costly, and are estimated to cost the US health care system $>$ \$12-17 billion annually [7]. In many countries health governors focus on unplanned postsurgical hospital readmissions as an objective metric for quality of care.

\section{Aim}

In this study, we aimed to determine the rate of readmissions after RIRS and PCNL operations, classify these factors, define the higher risk patients for readmission and develop prevention strategies.

\section{Material and methods}

A historical cohort study was conducted at two centers between 2013 and 2016. All patients who had an RIRS or PCNL operation during that period at these two institutions (Bahcesehir University and Medeniyet University, Istanbul, Turkey) were included in this study. A 7 Fr Karl Storz Flex X2 fiber optic flexible ureteroscope was used through a 9.5 Fr Cook ureteral access sheath and a holmium YAG laser for stone fragmentation in every RIRS. PCNL operations were made in the prone position, a $30 \mathrm{Fr}$ Amplatz sheath was used and stone fragmentation was done by both ultrasonic and pneumatic lithotripters. All patients were followed up with a urine analysis, total blood count, serum creatinine, non-contrast, urinary computed tomography (CT) scan for residual stones, readmissions and complications after the $30^{\text {th }}$ day following the procedure. Readmission is defined as an admission to a hospital within 30 days of a discharge. Patients who had concomitant surgery, any type of malignancy or who were hospitalized for other reasons before the procedure were not included in the study. All patients had sterile urine culture anal- ysis before surgery. All patients received antibiotic prophylaxis with second-generation cephalosporins before surgery and oral antibiotics were prescribed after the procedure. Post-operative JJ stenting was not a routine with the exception of suspected ureteral injury, large residual fragments, stones or obstruction. Stents were removed in due course, usually after 2 weeks, if there were no contraindications. ASA classifications were obtained through anesthesia records.

\section{Statistical analysis}

Categorical variables were analyzed by Pearson $\chi^{2}$ analysis, Fisher-Freeman-Halton and Student's $t$-test. The variables were first evaluated by univariate analysis. Given the differences in clinical and operative characteristics of the two groups included, all data were separately evaluated and compared. The ones with statistical significance or a close $p$-value to significance were included in a multivariate logistic regression analysis using Stata (version 12). A $p$-value $<0.05$ was considered to be significant.

\section{Results}

In this study a total of 471 patients were evaluated. All patients had renal stones in one kidney. The PCNL group consisted of 177 (37.6\%) patients, whereas the RIRS group had 294 (62.4\%) patients. The mean age of the patients was $44.59 \pm 16.34$ years, ranging from 2 to 82 years. The larger group (RIRS) had 294 (62.4\%) patients and they were all operated on by flexible ureteroscopy. The other group had 177 (37.6\%) patients who had a PCNL operation. Of the RIRS group, 60 (20.4\%) patients and 48 (27.1\%) patients of the PCNL group were readmitted. PCNL surgery was found to cause significantly higher risk for readmission when compared to RIRS ( $p=0.0041)$. Baseline characteristics and also the pre-operative, post-operative, intra-operative factors are shown in Table I.

The statistical analysis showed no significant correlation between readmission rates and age, ASA class, stone treatment prior to surgery, largest stone diameter or symptoms at diagnosis $(p>0.05)$.

Having multiple kidney stones was found to be an independent and statistically significant risk factor for readmission in both RIRS $(p=0.02)$ and PCNL $(p=0.01)$ groups.

There were 39 (22\%) complications in the PCNL group. Most of them (23) were intra-operative bleed- 
Table I. Patients' characteristics (pre-, intra-, post-operative and discharge factors)

\begin{tabular}{|c|c|c|c|c|}
\hline Parameter & Total $(n=471)$ & PCNL $(n=177)$ & $\operatorname{RIRS}(n=294)$ & $P$-value \\
\hline Age, mean \pm SD [years] & $44.59 \pm 16.34$ & $42.05 \pm 14.29$ & $46.12 \pm 17.30$ & ${ }^{\mathrm{a}} 0.006^{\star *}$ \\
\hline ASA class: & & & & b0.027* \\
\hline 1 & $416(88.3)$ & $152(85.9)$ & $264(89.8)$ & \\
\hline 2 & $51(10.8)$ & $21(11.9)$ & $30(10.2)$ & \\
\hline 3 & $4(0.8)$ & $4(2.3)$ & 0 & \\
\hline Prior stone therapy: & $273(58.0)$ & $64(36.2)$ & $209(71.1)$ & ${ }^{c} 0.001^{* *}$ \\
\hline ESWL & $80(17.0)$ & $18(10.2)$ & $62(21.1)$ & \\
\hline Ureteroscopy & $51(10.8)$ & $11(6.2)$ & 40 (13.6) & \\
\hline Open surgery & $32(6.8)$ & $13(7.3)$ & $19(6.5)$ & \\
\hline PCNL & $23(4.9)$ & $7(4.0)$ & $16(5.4)$ & \\
\hline Multi & $87(18.5)$ & $15(8.5)$ & $72(24.5)$ & \\
\hline Major symptom at diagnosis: & & & & ${ }^{c} 0.225$ \\
\hline Pain & $345(73.2)$ & $128(72.3)$ & $217(73.8)$ & \\
\hline Hematuria & $32(6.8)$ & $10(5.6)$ & $22(7.5)$ & \\
\hline Other urinary symptoms/infection & $58(12.3)$ & $20(11.3)$ & 38 (12.9) & \\
\hline Asymptomatic/Incidental & $36(7.6)$ & 19 (10.7) & $17(5.8)$ & \\
\hline Number of stones: & & & & ${ }^{\circ} 0.767$ \\
\hline Single & $185(39.3)$ & $68(38.4)$ & $117(39.8)$ & \\
\hline Multiple & $286(60.7)$ & $109(61.6)$ & $177(60.2)$ & \\
\hline Largest stone diameter [mm]: & & & & ${ }^{c} 0.001^{\star *}$ \\
\hline$<10$ & $68(14.4)$ & $2(1.1)$ & $66(22.4)$ & \\
\hline $10-20$ & $239(50.7)$ & $52(29.4)$ & $187(63.6)$ & \\
\hline$>20$ & $164(34.8)$ & $123(69.5)$ & $41(13.9)$ & \\
\hline Complications: & $60(12.7)$ & $39(22.0)$ & $21(7.1)$ & ${ }^{c} 0.001^{\star *}$ \\
\hline Ureteral injury & $4(0.8)$ & 0 & $4(1.4)$ & \\
\hline Bleeding causing transfusion & $23(4.9)$ & $21(11.9)$ & $2(0.7)$ & \\
\hline Sepsis & $22(4.7)$ & $9(5.1)$ & $13(4.4)$ & \\
\hline Other, explain & $9(1.9)$ & $9(5.1)$ & 0 & \\
\hline Respiratory (atelectasis, pneumonia) & $2(0.4)$ & 0 & $2(0.7)$ & \\
\hline Postoperative J stent placement: & & & & ${ }^{c} 0.001^{\star *}$ \\
\hline Yes & $300(63.7)$ & $30(16.9)$ & $270(91.8)$ & \\
\hline No & $171(36.3)$ & $147(83.1)$ & $24(8.2)$ & \\
\hline Residual stone/fragments: & & & & ${ }^{c} 0.001^{\star *}$ \\
\hline Yes & $183(38.9)$ & $96(54.2)$ & $87(29.6)$ & \\
\hline No & $288(61.1)$ & $81(45.8)$ & $207(70.4)$ & \\
\hline Prescription: & & & & ${ }^{\mathrm{b}} 0.001^{\star \star}$ \\
\hline No & $5(1.1)$ & 0 & $5(1.7)$ & \\
\hline Antibiotics & $34(7.2)$ & $16(9.0)$ & $18(6.1)$ & \\
\hline NSAID & $249(52.9)$ & $81(45.8)$ & $168(57.1)$ & \\
\hline Narcotic & $8(1.7)$ & $7(4.0)$ & $1(0.3)$ & \\
\hline Antibiotics + NSAID & $175(37.2)$ & $73(41.2)$ & $102(34.7)$ & \\
\hline
\end{tabular}

${ }^{a}$ Student's $t$ test, ${ }^{b}$ Fisher-Freeman-Halton Test, ${ }^{c}$ Pearson $\chi^{2}$ test, ${ }^{*} p<0.05,{ }^{* *} p<0.01$. 


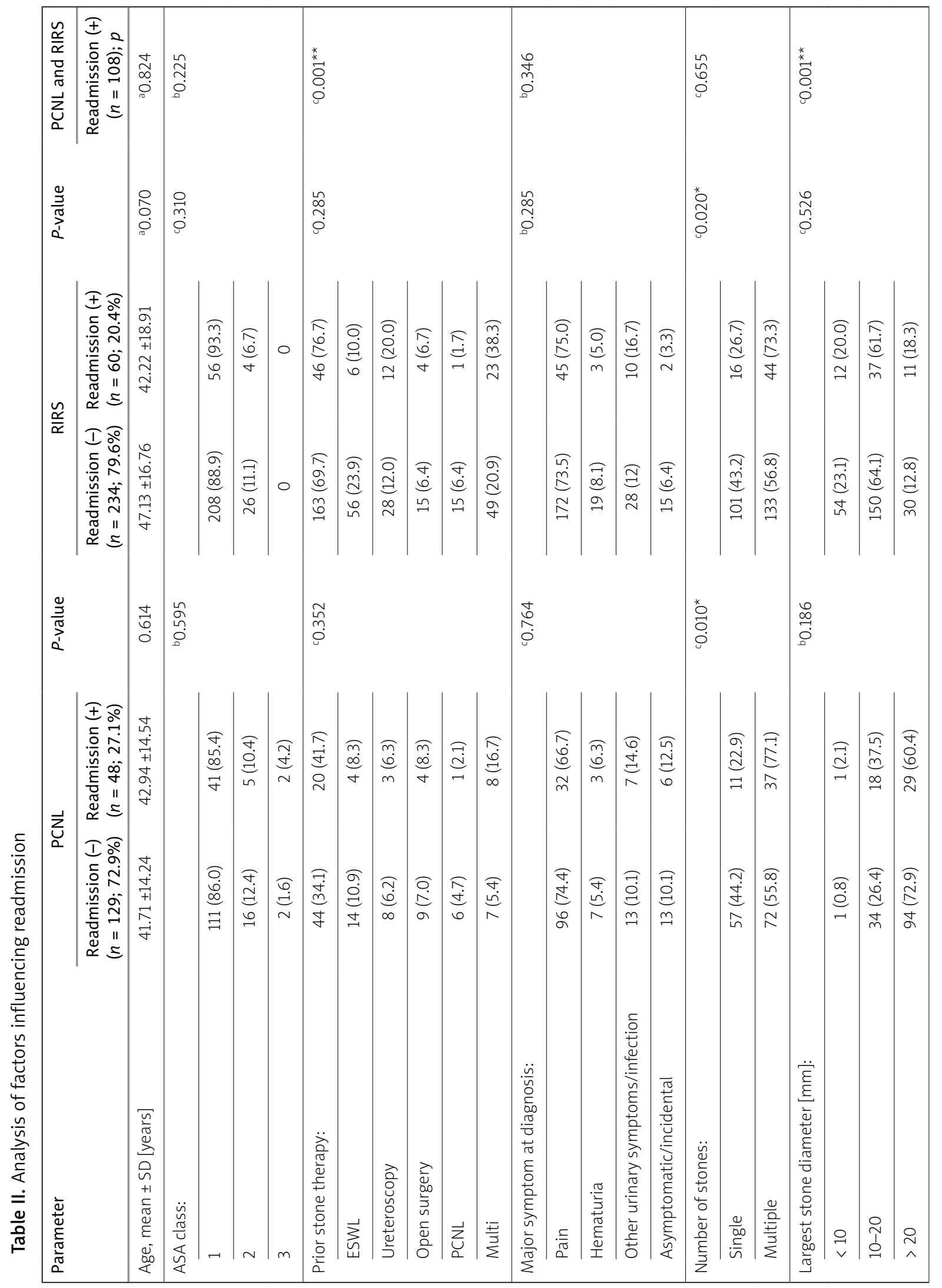




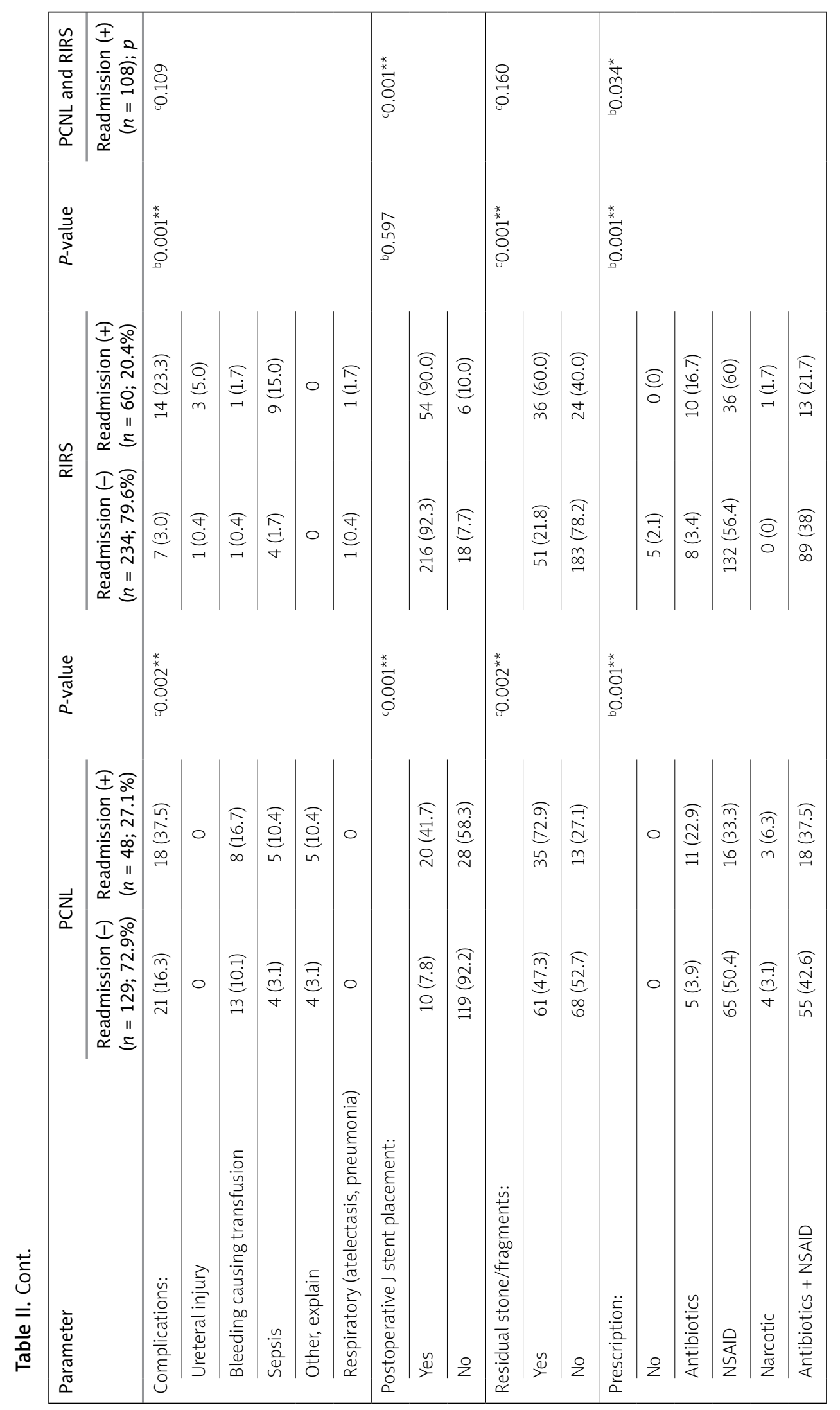


Table III. Reasons for readmission

\begin{tabular}{|lcc|}
\hline Parameter & PCNL & RIRS \\
\hline Uncontrolled pain & $20(11.2 \%)$ & $18(6.1 \%)$ \\
\hline Infection/sepsis & $17(9.6 \%)$ & $22(7.4 \%)$ \\
\hline Hematuria & $4(2.2 \%)$ & $3(1 \%)$ \\
\hline Urinary retention & 0 & $12(4 \%)$ \\
\hline J stent displacement & $7(3.9 \%)$ & $5(1.7 \%)$ \\
\hline Total & $48(27.1 \%)$ & $60(20.4 \%)$ \\
\hline
\end{tabular}

ing requiring blood transfusion. On the other hand, the RIRS group had 21 (7.1\%) complications. Perioperative complications were an important risk factor for early readmission in both RIRS $(p=0.001)$ and PCNL $(p=0.002)$ groups.

Postoperative JJ stent placement was found to be significantly correlated with readmission for the PCNL group $(p=0.001)$.

Residual stone was defined as fragments over $2 \mathrm{~mm}$ in size in the post-operative CT scan. Having residual stones after surgery was found to be a statistically significant risk factor for both groups ( $p=$ 0.002 and $p=0.001$ ).

The post-operative use of antibiotics was also found to be correlated with higher readmission for the PCNL group $(p=0.001)$.

The reasons and analysis of the readmission after surgery groups are shown in Tables II and III.

\section{Discussion}

To the best of our knowledge, there have not been a significant number of papers published in the literature investigating the readmission rates and the causative factors with regard to the two most common surgical techniques for kidney stones in the last decade. Although PCNL seems to have a higher risk for readmission, it should be considered that the numbers and characteristics of the two groups were not similar in this retrospective study. Therefore, rather than comparing these well-known surgical techniques with each other, we aimed to determine the factors influencing the readmission rate for both groups separately.

In 1997, the re-admission rate for day-case urological procedures was unacceptably high, 9.3\% [8]. A decade later, in 2007, Sinclair et al. reported that day-case urological surgery, despite its increasing frequency and the degree of complexity, had an acceptable and an improved overall readmission rate of 2.4\% [9]. Rapid technological advances as well as increasing experience of the urologists might be responsible for this phenomenon.

There have been some studies investigating the readmission rates for ambulatory procedures in urology. Rambachan et al. [10] suggested that readmission after outpatient urological surgery occurs at a rate of $3.7 \%$, which is fourth of the 10 tracked surgical specialties. These results represent many endoscopic procedures, including minimally invasive ones, rendering our data comparable with those from the aforementioned studies [10, 11].

In 2016 Bloom et al. reported their data related to readmissions following elective ureteroscopy. They found a $5.8 \%$ readmission rate, which is similar to our RIRS results. The authors also suggested that the comorbidities, particularly chronic obstructive pulmonary disease (COPD) and hypertension, were significantly correlated with the readmission rate after ureteroscopy. We did not investigate these variables individually, but the ASA score was not found to be a risk factor for readmission in our cohort [12].

Higher stone burden was found to be a significant risk for readmission. This can be explained by both the longer time of the operation and also the higher rates of residual stones or fragments. A number of patients had been followed for asymptomatic stones. Nevertheless, possible future events are the subject of investigation in these patients as well. In 2015, Selby and colleagues suggested that total stone volume is the optimal method of quantifying stone burden for the purpose of predicting future stone events. The authors recommended a baseline CT scan in stone formers for risk stratification [13]. We encourage future studies investigating the predictive tools for asymptomatic kidney stones which tend to become symptomatic afterwards.

If the patients with a lower stone burden are elected for surgery before they become symptomatic, readmission rates are expected to be lower subsequently.

The use of antibiotic prophylaxis to prevent urinary tract infection and sepsis following endoscopic urological procedures is controversial [14, 15]. In this study, despite antibiotic prophylaxis with second generation cephalosporins in all patients, 39 patients were readmitted because of urinary infection or sepsis (Table III). Thirteen (30\%) of them 
had previous stone treatments and urinary infections. However, this association did not reach a statistical significance. These patients may deserve prophylaxis with broad spectrum antibiotics even if they do not have a proven infection prior to the operation.

Postoperative stenting after an uneventful ureteroscopy is controversial $[16,17]$. It was suggested by Torricelli et al. after flexible URS via a ureteral access sheath, to decrease post-operative complications and pain [18]. In our study, post-operative JJ stent placement was a significant risk factor for readmission for the PCNL group, being somewhat contrary to the other studies. However, in our series, an increase of the readmission rate may not be completely attributed to the stenting itself, considering the fact that postoperative stenting was the procedure of choice in already high-risk patients with residual stones, obstruction or ureteric injury. As it stands, the study has some limitations due to its retrospective nature and limited number of patients. The question whether routine stenting after PCNL or RIRS is necessary or it should be reserved for highrisk patients remains unanswered and warrants further comparative randomized studies.

\section{Conclusions}

Stone burden and residual stone/fragments are the most important factors for readmissions after PCNL or RIRS operations. Patients who had previous stone treatments and urinary infections are predisposed to post-operative urinary infections. A prospective, randomized trial may be useful to determine risk factors for readmission after kidney stone surgery.

\section{Conflict of interest}

The authors declare no conflict of interest.

\section{References}

1. Cho SY. Current status of flexible ureteroscopy in urology. Korean J Urol 2015; 56: 680-8.

2. Raheem OA, Mirheydar HS, Miller DL et al. Contemporary trends in the ambulatory surgical treatment of urolithiasis: population-based analysis. J Endourol 2015; 29: 1189-92.

3. Chung HS, Jung SI, Yu HS et al. Modified totally tubeless percutaneous nephrolithotomy: is it an effective and safe treatment option for renal and upper ureteral stones? Videosurgery Miniinv 2016; 11: 240-6.

4. Lahme S. Miniaturisation of PCNL. Urolithiasis 2018; 46: 99-106.
5. Kim BS. Recent advancement or less invasive treatment of percutaneous nephrolithotomy. Korean J Urol 2015; 56: 614-23.

6. Turk C, Petrik A, Sarica K et al. EAU guidelines on interventional treatment for urolithiasis. Eur Urol 2016; 69: 475-82.

7. Jencks SF, Williams MV, Coleman EA. Rehospitalizations among patients in the medicare fee-for-service program. Reply. N Engl J Med 2009; 361: 312.

8. Crew JP, Turner KJ, Millar J, et al. Is day case surgery in urology associated with high admission rates? Ann Roy Coll Surg 1997; 79: 416-9.

9. Sinclair AM, Gunendran T, Pearce I. Day-case urological surgery: are we improving? BJU Int 2007; 99: 491-3.

10. Rambachan A, Matulewicz RS, Pilecki M, et al. Predictors of readmission following outpatient urological surgery. J Urol 2014; 192: 183-8.

11. Paez A, Redondo E, Linares A, et al. Adverse events and readmissions after day-case urological surgery. Int Braz J Urol 2007; 33: 330-8.

12. Bloom J, Matthews G, Phillips J. Factors influencing readmission after elective ureteroscopy. J Urol 2016; 195: 1487-91.

13. Selby MG, Vrtiska TJ, Krambeck AE, et al. Quantification of asymptomatic kidney stone burden by computed tomography for predicting future symptomatic stone events. Urology 2015; 85 : 45-50.

14. Alsaywid BS, Smith GH. Antibiotic prophylaxis for transurethral urological surgeries: systematic review. Urol Ann 2013; 5: 61-74.

15. Pricop C, Dorobat C, Puia D, et al. Antibiotic prophylaxis in retrograde ureteroscopy: what strategy should we adopt? Germs 2013; 3: 115-21.

16. Wu Z, Ding Q, Jiang HW, et al. Routine ureteral stenting after ureteroscopic lithotripsy is not required: a prospective randomized trial. J Endourol 2007; 21: A255-A.

17. Srivastava A, Gupta R, Kumar A, et al. Routine stenting after ureteroscopy for distal ureteral calculi is unnecessary: results of a randomized controlled trial. J Endourol 2003; 17: 871-4.

18. Torricelli FC, De SB, Hinck B, et al. Flexible ureteroscopy with a ureteral access sheath: when to stent? Urology 2014; 83: 278-81.

Received: 25.06.2018, accepted: 28.07.2018. 